\title{
Unsteady Viscous Dissipative Dusty Nanofluid Flow Over a Vertical Plate
}

\author{
D.R.V.S.R.K. Sastry ${ }^{\# 1}$, V. Venkataraman ${ }^{\# 2}$, K. Kannan ${ }^{\# 3}$, M.Srinivasu ${ }^{* 4}$ \\ \# Department of Mathematics, SASTRA University, Tirumalaisamudram, Thanjavur, Tamil Nadu, India \\ ${ }^{1}$ drvsrksastry@gmail.com \\ 2 mathvvr@maths.sastra.edu \\ ${ }^{3}$ kkannan@maths.sastra.edu \\ * Department of Mathematics, Aditya College of Engineering, Surampalem, Andhra Pradesh, India \\ ${ }^{4}$ msr.mathematics@gmail.com
}

\begin{abstract}
The flow past an infinite vertical isothermal plate started impulsively in its own plane in a viscous incompressible two-phase nanofluid has been considered by taking into account the viscous dissipative heat. Two nano particles Copper $(\mathrm{Cu})$ and Alumina $\left(\mathrm{Al}_{2} \mathrm{O}_{3}\right)$ are submerged in a base fluid, Water $\left(\mathrm{H}_{2} \mathbf{0}\right)$. The coupled non-linear partial differential equations which govern the flow are solved for nanofluid and dust particle phases by finite difference method. The velocity and temperature fields have been shown graphically for various parameters. Here Grashof number, (Gr) being positive (cooling of the plate) for dusty air. Also the effects of Eckert number on heat transfer and skin friction coefficient for various parameters are represented graphically. It is observed that dusty nanofluid enhances both skin friction and heat transfer rate in the case of cooling.
\end{abstract}

Keyword-Free convection, Nanofluid, Viscosity, Two-phase flow, Dust particles

\section{INTRODUCTION}

Numerous theoretical and experimental studies of heat transfer induced by convection in fluids of porous and non-porous medium are studied by several researchers ([1]-[5]). These studies have many applications in physical systems like nuclear reactors, combustion systems, pneumatic transport etc. The study of the flow of dusty fluids is of practical importance, particularly, through packed beds, sedimentation, environmental pollution, chemical reactors, combustion systems, pneumatic transport and centrifugal separation of particles. In the filtration process and drying of porous materials in textile industries, Stokes problem on free convection effects is used. The stability of a laminar flow is studied by investigating the effects of dust particles on the critical Reynolds number [6].

The fluid flow and heat transfer over various geometrical surfaces have wide range of applications in engineering and its allied areas. Since low thermal conductivity in convectional fluids such as water, ethylene glycol, and oil has encountered variety of problems in engineering electronic devices, many researchers concentrated on nanofluids, to overcome this drawback and to enhance the thermal conductivity in the convectional fluids. Mixing of nano meter sized particles into base fluid is called nanofluid, which helps to enhance the thermal conductivity of the mixture fluid. Also mixing of micrometer sized particles (dust particles) in the base fluids improve the thermal conductivity of the base fluid and is called dusty fluid. Till now, researchers concentrated on investigating the momentum and heat transfer behaviour of either dusty or nanofluid particles. Magneto hydrodynamic flow of a viscous and elastic fluid embedded with dust particles over aligned parallel plates is discussed [7]. Momentum and heat transfer characteristics of MHD flow over a stretching sheet is analyzed [8]. Convection in an unsteady dusty fluid over rectangular channel is studied [9]. Dusty viscous fluid flow through naturally occurred porous medium is presented [10]. Dusty viscous fluid between two infinite parallel plates by considering aligned magnetic field is discussed [11]. The boundary layer flow of a nanofluid over a stretching sheet by considering convective boundary conditions is analyzed [12]. Study on the radiation effect on viscous nanofluid flow over a nonlinear stretching sheet is observed [13].

Numerical solution of stokes problem for free convection effects in dissipative dusty medium is studied and noticed how Grashof and Eckert numbers influence dusty fluid flow [14]. In this paper, we extended the work to nanofluids and analyzed the temperature, velocity and heat transfer characteristics along with various parameters. The current results are in good agreement with the results presented in [14].

\section{FORMULATION OF THE PROBLEM}

Consider a viscous unsteady incompressible two-phase nanofluid flow past an infinite vertical isothermal plate started impulsively in its own plane by taking into account the viscous dissipative heat. Two nano particles Copper $\left(\mathrm{C}_{u}\right)$ and Alumina $\left(\mathrm{Al}_{2} \mathrm{O}_{3}\right)$ are submerged in a base fluid Water $\left(\mathrm{H}_{2} \mathrm{O}\right)$. The $x^{\prime}$-axis is taken along the infinite plate in vertical direction and the $y^{\prime}$-axis is taken normal to the plate. The temperature of the plate is the same as that of the fluid when $t^{\prime}<0$. The plate starts at rest and moves in its own plane with a velocity $\mathrm{u}_{0}$ at 
$t^{\prime}>0$ and the temperature is instantaneously raised or lowered to a constant temperature $T_{w}^{\prime}$. It is also taken into account that all the fluid properties are assumed to be constant except that the influence of density variation with temperature has been considered only in the body force term. The buoyancy force on the dust particles is neglected. It is further neglected the volume fraction and viscosity of the pseudo fluid of dust particles. The set of governing equations of motion modelled in [6] for dusty fluid is extended to nanofluid as follows.

Equation of Continuity:

$$
\frac{\partial u^{\prime}}{\partial x^{\prime}}+\frac{\partial u^{\prime}}{\partial y^{\prime}}=0 \text { and } \frac{\partial u_{p}^{\prime}}{\partial x^{\prime}}+\frac{\partial u_{p}^{\prime}}{\partial y^{\prime}}=0
$$

Equation of Momentum:

$$
\begin{aligned}
& \rho_{n f} \frac{\partial u^{\prime}}{\partial t^{\prime}}=\mu_{n f} \frac{\partial^{2} u^{\prime}}{\partial y^{\prime 2}}+g \beta\left(T^{\prime}-T_{\infty}^{\prime}\right)+K N_{0}\left(u_{p}^{\prime}-u^{\prime}\right) \\
& \frac{\partial u_{p}^{\prime}}{\partial t^{\prime}}=-\frac{\left(u_{p}^{\prime}-u^{\prime}\right)}{\tau_{p}}
\end{aligned}
$$

Equation of Energy:

$$
\begin{aligned}
& \left(\rho c_{p}\right)_{n f} \frac{\partial T^{\prime}}{\partial t^{\prime}}=k_{n f} \frac{\partial^{2} T^{\prime}}{\partial y^{\prime 2}}+\frac{N_{0} m c_{s}}{\tau_{T}}\left(T_{p}^{\prime}-T^{\prime}\right)+\mu_{n f}\left(\frac{\partial u^{\prime}}{\partial y}\right)^{2} \\
& \frac{\partial T_{p}^{\prime}}{\partial t^{\prime}}=-\frac{\left(T_{p}^{\prime}-T^{\prime}\right)}{\tau_{T}}
\end{aligned}
$$

where $u^{\prime}, u_{p}^{\prime}$ are velocities of the fluid and dust particles respectively, $g$ is acceleration due to gravity, $\beta$ is coefficient of volume expansion. The temperature of fluid and dust particles are denoted by $T^{\prime}, T_{p}^{\prime}$ respectively. Further $T_{w}^{\prime}, T_{\infty}^{\prime}$ are wall and ambient temperatures respectively. Also, one can write the relaxation time during which the velocity of the particle phase relative to the fluid is reduced $(1 / e)$ times its initial value, $\tau_{p}=m / 6 \pi \mu r_{p}$ and thermal relaxation time of particle phase, $\tau_{T}=m c_{s} / 4 \pi K r_{p}$ where $m$ is mass of each dust particle, $c_{s}$ is specific heat of dust particle, $N_{0}$ is number density of dust particle, $K$ is Stokes resistance coefficient. Further if $\phi$ is the solid nanoparticles volume fraction, then the effective dynamic viscosity, density, heat capacitance, thermal conductivity of the nanofluid are defined respectively as

$$
\begin{aligned}
& \mu_{n f}=\frac{\mu_{f}}{(1-\varphi)^{2.5}} ; \rho_{n f}=(1-\phi) \rho_{f}+\phi \rho_{s} ;\left(\rho C_{p}\right)_{n f}=(1-\phi)\left(\rho C_{p}\right)_{f}+\phi\left(\rho C_{p}\right)_{s} \\
& \text { and } \mathrm{k}_{\mathrm{nf}}=\mathrm{k}_{\mathrm{f}}\left(\frac{\mathrm{k}_{\mathrm{s}}+2 \mathrm{k}_{\mathrm{f}}-2 \phi\left(\mathrm{k}_{\mathrm{f}}-\mathrm{k}_{\mathrm{s}}\right)}{\mathrm{k}_{\mathrm{s}}+2 \mathrm{k}_{\mathrm{f}}+\phi\left(\mathrm{k}_{\mathrm{f}}-\mathrm{k}_{\mathrm{s}}\right)}\right)
\end{aligned}
$$

The initial and boundary conditions of the problem may be defined as

for $t^{\prime} \leq 0, \quad u^{\prime}=u_{p}^{\prime}=0, T^{\prime}=T_{p}^{\prime}=T_{\infty}^{\prime}$

for $t^{\prime}>0, u^{\prime}=u_{0}, T^{\prime}=T_{w}^{\prime}$ at $y^{\prime}=0$ and $u^{\prime} \rightarrow 0, T^{\prime} \rightarrow T_{\infty}^{\prime}$ as $y^{\prime} \rightarrow \infty$

Using non dimensional variables,

$$
y=\frac{u_{0} y^{\prime}}{v_{f}}, \quad u=\frac{u^{\prime}}{u_{0}}, \quad u_{p}=\frac{u_{p}^{\prime}}{u_{0}}, \quad \theta=\frac{T^{\prime}-T_{\infty}^{\prime}}{T_{w}^{\prime}-T_{\infty}^{\prime}}, \theta_{p}=\frac{T_{p}^{\prime}-T_{\infty}^{\prime}}{T_{w}^{\prime}-T_{\infty}^{\prime}}, \quad t=\frac{t^{\prime} u_{0}^{2}}{v_{f}}
$$

One can transform the governing equations of motion given in (2) - (5) as the following non-dimensional partial differential equations

$$
\frac{\partial u}{\partial t}=\lambda_{1} \lambda_{2} \frac{\partial^{2} u}{\partial y^{2}}+G_{r} \theta+\lambda_{2} \frac{\alpha_{1}}{\alpha_{2}}\left(u_{p}-u\right)
$$




$$
\begin{aligned}
& \frac{\partial u_{p}}{\partial t}=-\frac{1}{\alpha_{2}}\left(u_{p}-u\right) \\
& \operatorname{Pr} \frac{\alpha_{3}}{\lambda_{3}} \frac{\partial \theta}{\partial t}=\frac{\partial^{2} \theta}{\partial y^{2}}+\frac{2}{3} \frac{\alpha_{1}}{\alpha_{2} \lambda_{3}}\left(\theta_{p}-\theta\right)+\frac{E c \operatorname{Pr} \lambda_{1}}{\lambda_{3}}\left(\frac{\partial u}{\partial y}\right)^{2} \\
& \gamma \operatorname{Pr} \frac{\partial \theta_{p}}{\partial t}=-\frac{2}{3 \alpha_{2}}\left(\theta_{p}-\theta\right)
\end{aligned}
$$

where $v_{f}$ is the kinematic viscosity of the base fluid, $G_{r}=\frac{v_{f} g \beta\left(T_{w}^{\prime}-T_{\infty}^{\prime}\right)}{u_{0}^{3}}$ (Grashof number) $\alpha=\frac{N_{0} m}{\rho_{f}}$ (concentration parameter), $\lambda=\frac{\tau_{p} u_{0}^{2}}{v_{f}}$ (non-dimensional relaxation time), $\tau_{p}=\frac{m}{K}$ ( $K=6 \pi \mu r_{p}, r_{p}$ is radius of the dust particle), $P_{r}=\frac{v_{f}\left(\rho c_{p}\right)_{f}}{k_{f}}$ (Prandtl number), $\lambda_{1}=\frac{1}{(1-\phi)^{2.5}}$, $\lambda_{2}=\frac{1}{\left(1-\phi+\phi \frac{\rho_{s}}{\rho_{f}}\right)} \quad, \quad \lambda_{3}=\frac{k_{s}+2 k_{f}-2 \phi\left(k_{f}-k_{s}\right)}{k_{s}+2 k_{f}+\phi\left(k_{f}-k_{s}\right)} \quad, \quad \gamma=\frac{c_{s}}{c_{p}} \quad \alpha_{2}=\frac{\tau_{p} u_{0}^{2}}{v_{f}} \quad$, $\alpha_{3}=(1-\phi)+\phi \frac{\left(\rho c_{p}\right)_{s}}{\left(\rho c_{p}\right)_{f}}$ and $E c=\frac{u_{0}{ }^{2}}{c_{p}\left(T_{w}^{\prime}-T_{\infty}^{\prime}\right)}$ (Eckert number). Also from the equations (6) and (7), one can express the initial and boundary conditions in terms of non-dimensional quantities as follows.

for $t \leq 0: u(y, t)=u_{p}(y, t)=0=\theta(y, t)=\theta_{p}(y, t)$

for $t>0: u(0, t)=u_{p}(0, t)=1=\theta(0, t)=\theta_{p}(0, t)$ and

$$
u(\infty, t)=u_{p}(\infty, t)=0=\theta(\infty, t)=\theta_{p}(\infty, t)
$$

\section{SOLUTION PROCEDURE}

The transformed equations of motion (8) - (11) along with the initial and boundary conditions (12a)and (12b) are solved by finite difference method. The partial differential equation coefficients are replaced by their finite difference quotients forming the following finite difference equations.

$$
\begin{aligned}
& \left(\frac{u_{i, j+1}-u_{i, j}}{\Delta t}\right)=\operatorname{Gr} \theta_{i, j}+\lambda_{1} \lambda_{2}\left(\frac{u_{i+1, j}-2 u_{i, j}+u_{i-1, j}}{(\Delta y)^{2}}\right)+\frac{\lambda_{2} \alpha_{1}}{\alpha_{2}}\left(u_{p_{(i, j)}}-u_{i, j}\right) \\
& \left(\frac{u_{p(i, j+1)}-u_{p(i, j)}}{\Delta t}\right)=-\frac{1}{\alpha_{2}}\left(u_{p(i, j)}-u_{(i, j)}\right) \\
& \operatorname{Pr} \cdot\left(\frac{\alpha_{3}}{\lambda_{3}}\right)\left(\frac{\theta_{(i, j+1)}-\theta_{(i, j)}}{\Delta t}\right)=\left(\frac{\theta_{(i+1, j)}-2 \theta_{(i, j)}+\theta_{(i-1, j)}}{(\Delta y)^{2}}\right) \\
& +\operatorname{Pr} \cdot E c \cdot\left(\frac{\lambda_{1}}{\lambda_{3}}\right)\left(\frac{u_{(i, j)}-u_{(i-1, j)}}{\Delta y}\right)^{2}+\frac{2 \alpha_{1}}{3 \alpha_{2} \lambda_{3}}\left(\theta_{p(i, j)}-\theta_{(i, j)}\right) \\
& \gamma \cdot \operatorname{Pr}\left(\frac{\theta_{p(i, j+1)}-\theta_{p(i, j)}}{\Delta t}\right)=-\left(\frac{2}{3 \alpha_{2}}\right)\left(\theta_{p(i, j)}-\theta_{(i, j)}\right)
\end{aligned}
$$

where the index i refers to $y$ and $\mathrm{j}$ refers to t. $\Delta y$ is taken to be 0.1. From the condition (12a), 
$u(0,0)=u_{p}(0,0)=1=\theta(0,0)=\theta_{p}(0,0)$ and $u(i, 0)=u_{p}(i, 0)=0=\theta(i, 0)=\theta_{p}(i, 0)$ for all $i \neq 0$

From the boundary conditions (12b), we get $u(0, j)=u_{p}(0, j)=1=\theta(0, j)=\theta_{p}(0, j)$ for all $j$.

According to the numerical solutions [14], it may be taken $y=4.1$ as the point corresponding to $y=\infty$. Therefore one may get $u(41, j)=u_{p}(41, j)=0=\theta(41, j)=\theta_{p}(41, j)$, which is true for all $j$ equivalent to the boundary condition (12b). The velocities at the end of the time step namely $u_{i, j+1}$ and $u_{p(i, j+1)}, i=1,40$ are computed from (13) and (14) in terms of velocities and temperatures at points of the earlier time step. Similarly $\theta_{i, j+1}, \theta_{p(i, j+1)}$ are computed from (15) and (16). The procedure is repeated till $t=1 \quad(j=400)$. The computations are carried out and the curves of velocity and temperature against $y$ are traced using MATLAB software.

\section{RESULTS AND DISCUSSION}

The velocity and temperature profiles are drawn for Prandtl number 6.785(Water) and time $t=0.2$. Thermo physical properties of nanoparticles [15] are given in Table1. The velocity profiles of both fluid and dust particle phases for different values of Grashof number are depicted in fig.1and fig.2. In the case of the plate being cooled by free convection currents we observe that due to presence of viscous dissipative heat, there is a rise not only in velocity of fluid(nanofluid) but also in dust particle phase. Also it follows from the figures that greater cooling of the plate causes a rise in velocity. Fig.3 and fig.4 explain how the Eckert number along with solid volume fraction influences the temperature profiles of both fluid and dust particle phases. In both the figures it is observed that there is a decrease in the thermal boundary layer with respect to increase in the volume fraction. Also in the case of cooling $(\mathrm{Gr}>0)$, greater viscous dissipative heat causes a rise in temperature not only in fluid but also in dust particles phase. The velocity profiles of both the phases are displayed in fig.5 for both the nanoparticles at different Grashof number. It is noted that compared to fluid velocity, dust particle velocity is less in both the nanoparticles. Also Copper nanoparticles move with more velocity than that of Alumina. From fig.6, it is observed that in the case of cooling, Alumina particles have high temperature profile than Copper. This variation is significant at low viscous dissipation. The variation of velocity profiles of both fluid and dust particle phase can be seen in fig.7 and fig.8 for two different values of a slightly higher mass concentration parameter. It is noticed that velocity increases with the increase of concentration parameter. We see a significant change in the base fluid $(\phi=0)$. The same is observed in both velocity profiles. Fig.9 shows the impact of Eckert number on heat transfer rate. The curves are drawn for two different values of mass concentration of dust particle's parameter. We notice that heat transfer insignificantly enhances with a small increase in the Eckert number. At higher mass concentration, the variation in the heat transfer rate is more. This is due to the fact that increasing dust particles will enhance the heat transfer in the fluid. In the similar way a plot is drawn for coefficient of skin friction against Eckert number which is shown in fig.(10). With the increase in time, the skin friction coefficient decreases. Also with small increase in Eckert number there is no much difference in the profile.

TABLE 1. Thermo Physical Properties of Nanoparticles

\begin{tabular}{|c|c|c|c|}
\hline Physical property & Pure Water & $\mathrm{Cu}$ & $\mathrm{Al}_{2} \mathrm{O}_{3}$ \\
\hline$\rho\left(\mathrm{kg} / \mathrm{m}^{3}\right)$ & 997.1 & 8933 & 3970 \\
\hline $\mathrm{C}_{\mathrm{p}}(\mathrm{J} / \mathrm{kg} \mathrm{K})$ & 4179 & 385 & 765 \\
\hline $\mathrm{k}(\mathrm{W} / \mathrm{m} \mathrm{K})$ & 0.613 & 401 & 40 \\
\hline
\end{tabular}




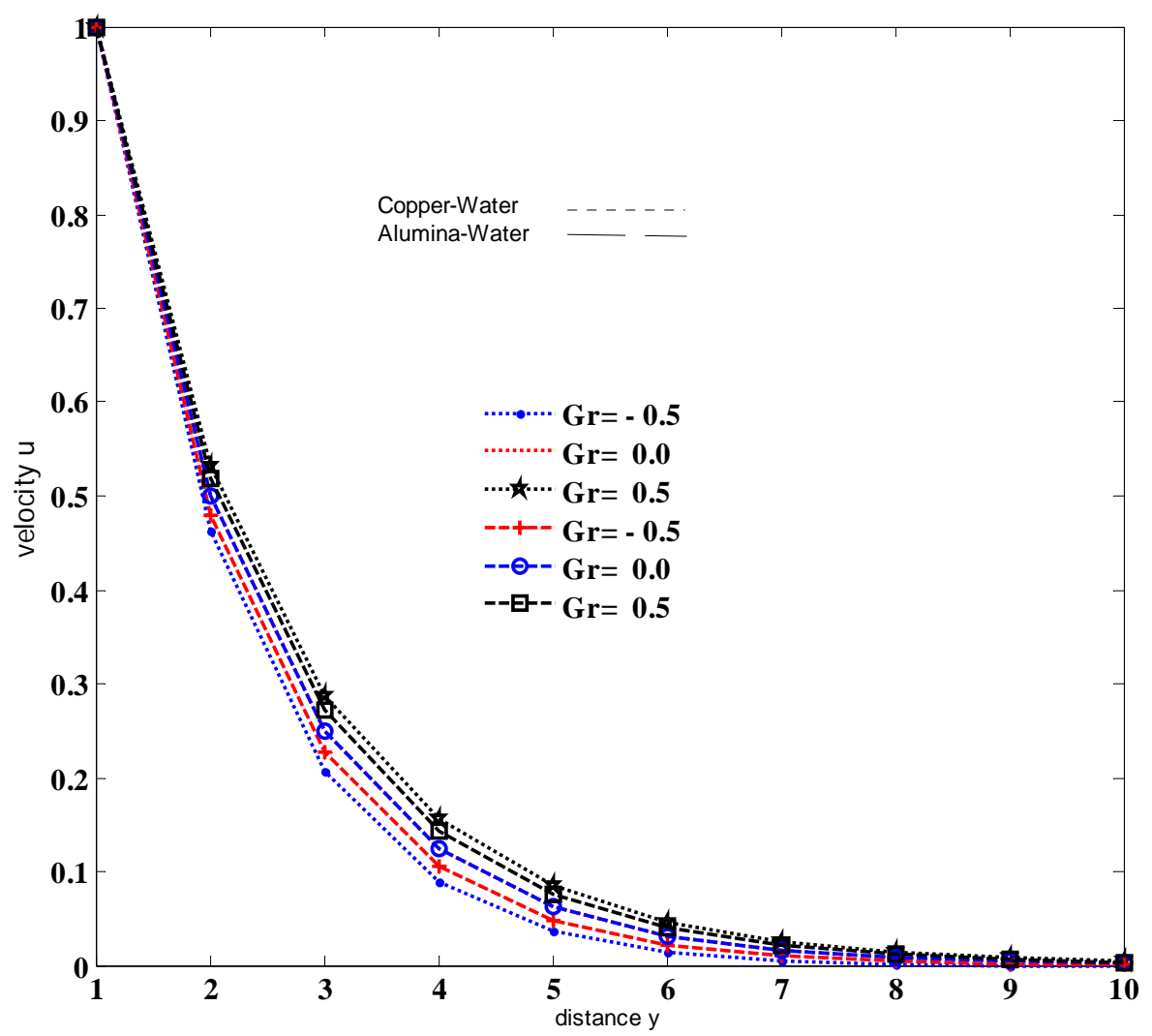

Fig.1. Velocity Profile for Fluid Phase $\left(E c=0.1, \alpha_{1}=0.0025, \alpha_{2}=0.025, \gamma=0.8, \phi=0.2\right)$

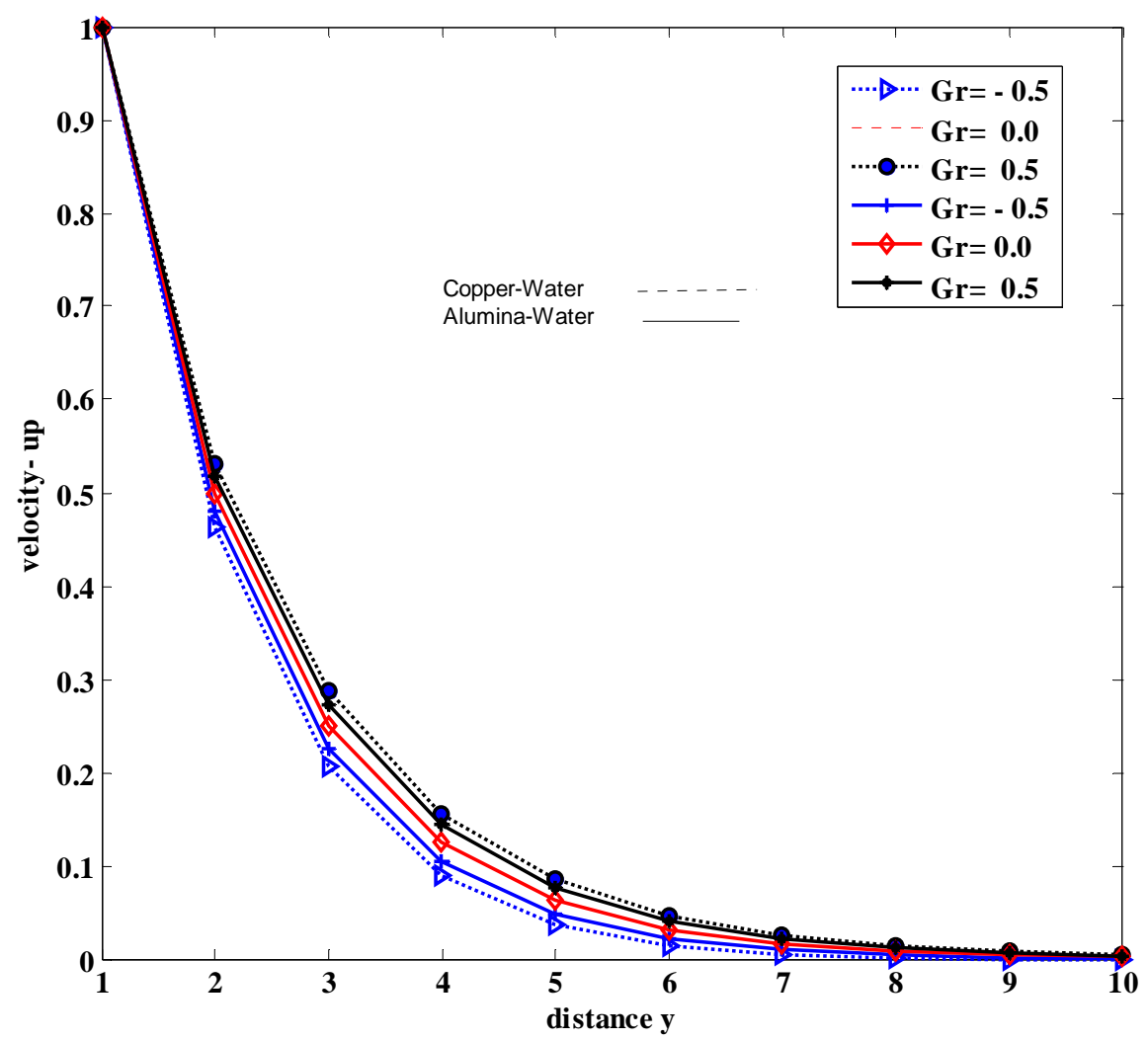

Fig.2. Velocity Profile for Dust Particle Phase $\left(E c=0.1, \alpha_{1}=0.0025, \alpha_{2}=0.025, \gamma=0.8, \phi=0.2\right)$ 


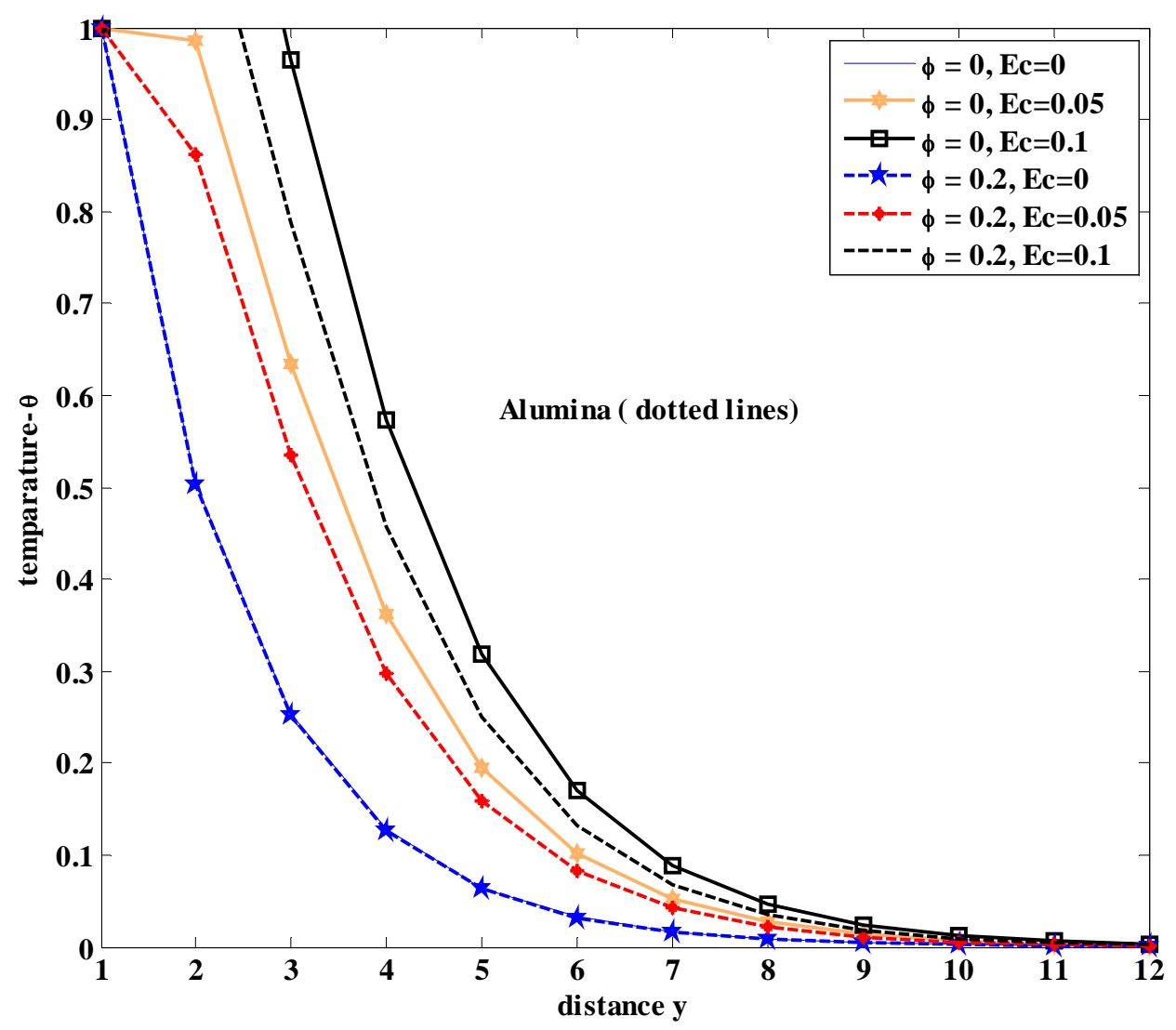

Fig.3. Temperature Profile for Fluid Phase $\left(G r=0.2, \alpha_{1}=0.0025, \alpha_{2}=0.025, \gamma=0.8\right)$

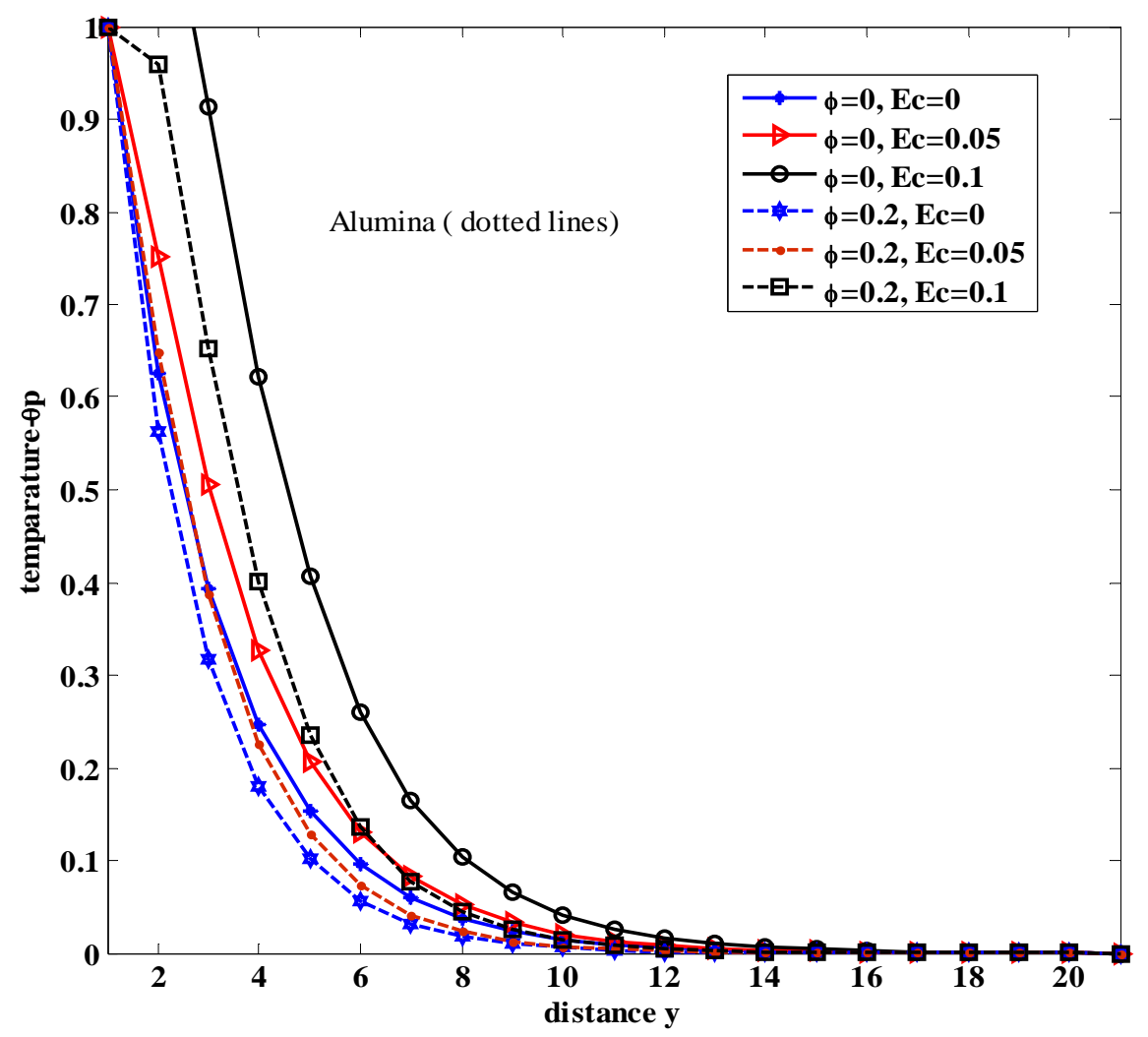

Fig.4. Temperature Profile for Dust Particle Phase $\left(G r=0.2, \alpha_{1}=0.0025, \alpha_{2}=0.025, \gamma=0.8\right)$ 


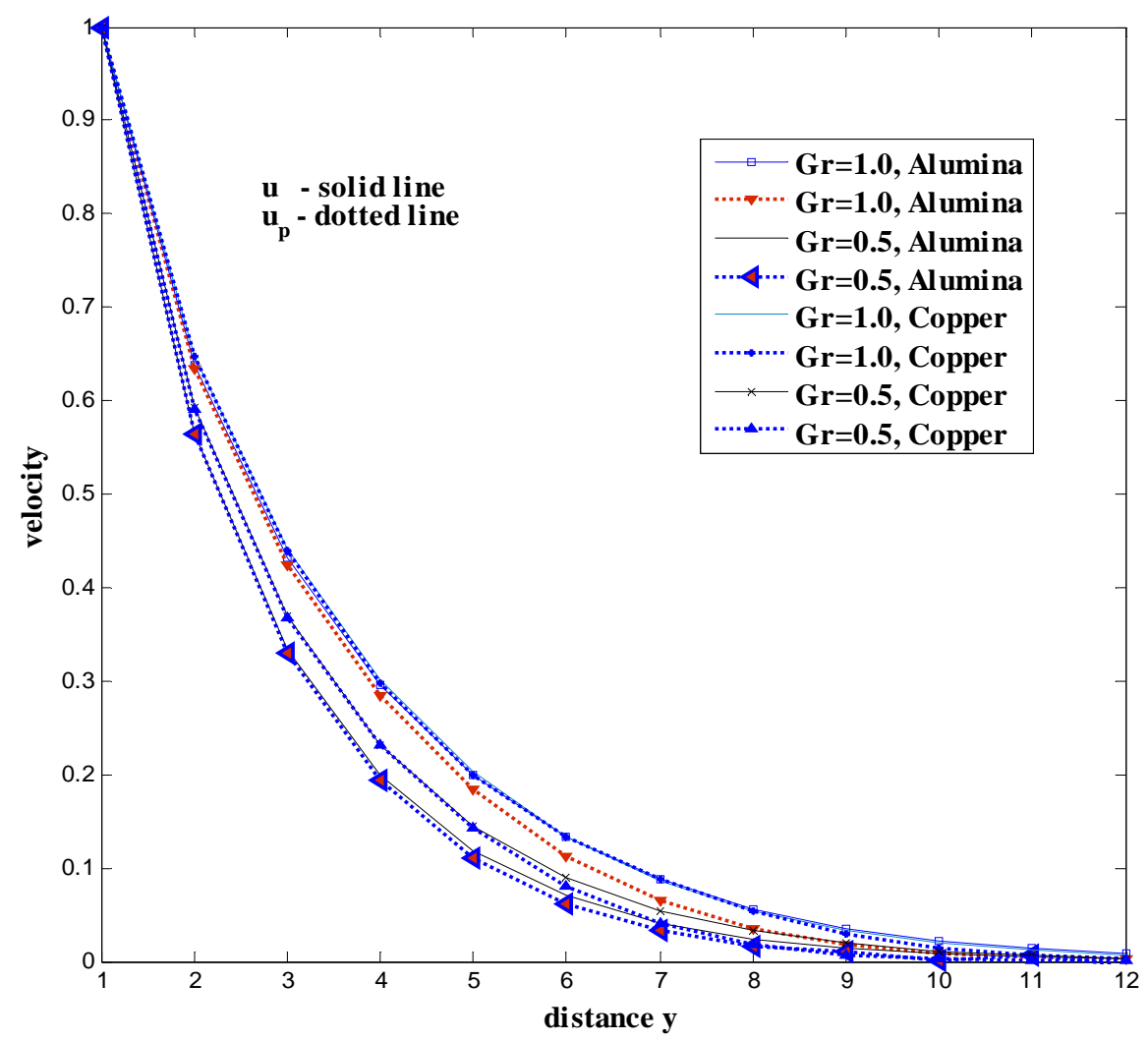

Fig.5. Velocity Profile for both Phases $\left(E c=0.1, \alpha_{1}=0.0025, \alpha_{2}=0.025, \gamma=0.8, \phi=0.2\right)$

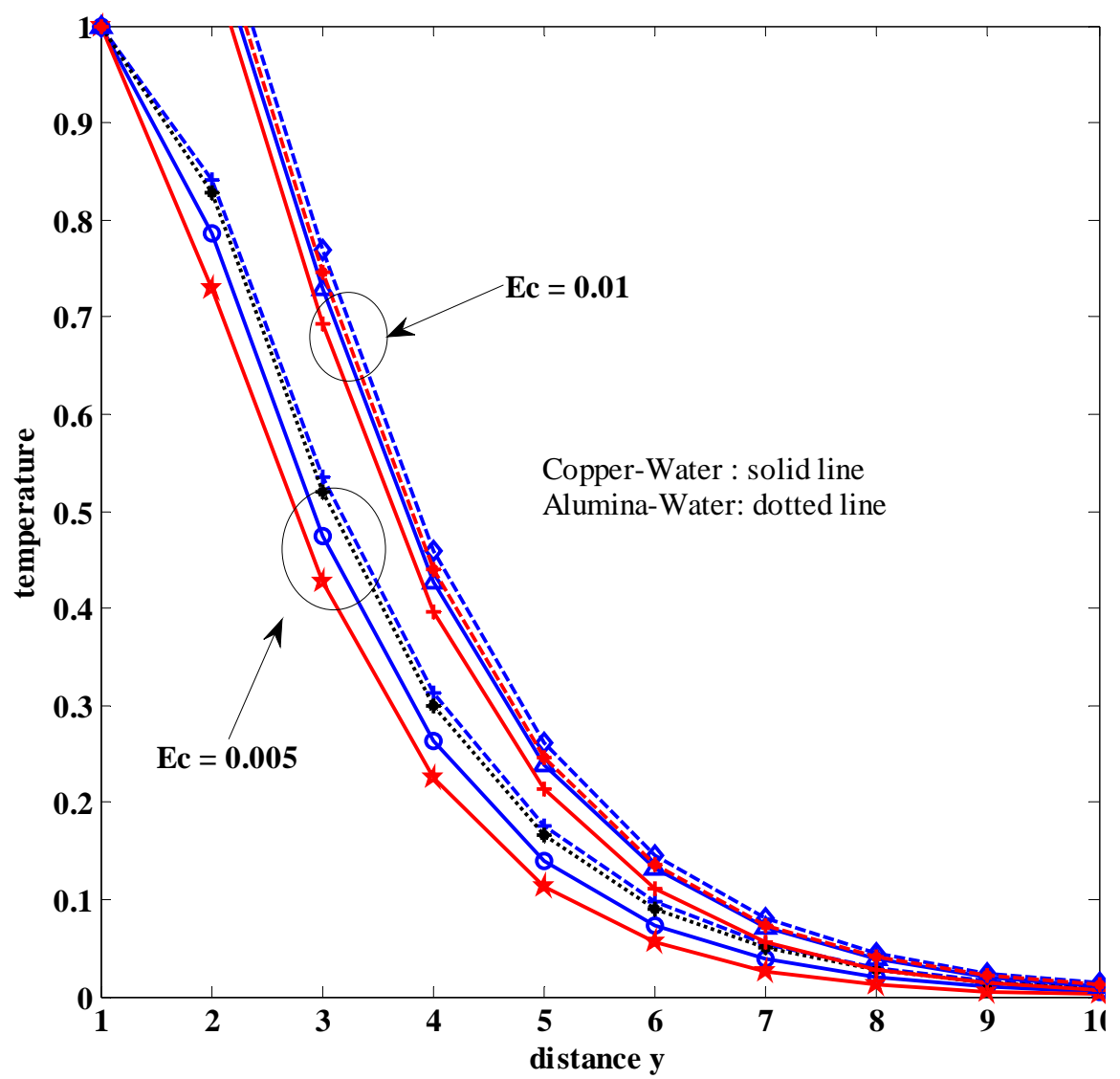

Fig.6. Temperature Profile for both Phases $\left(G r=0.2, \alpha_{1}=0.0025, \alpha_{2}=0.025, \gamma=0.8, \phi=0.2\right)$ 


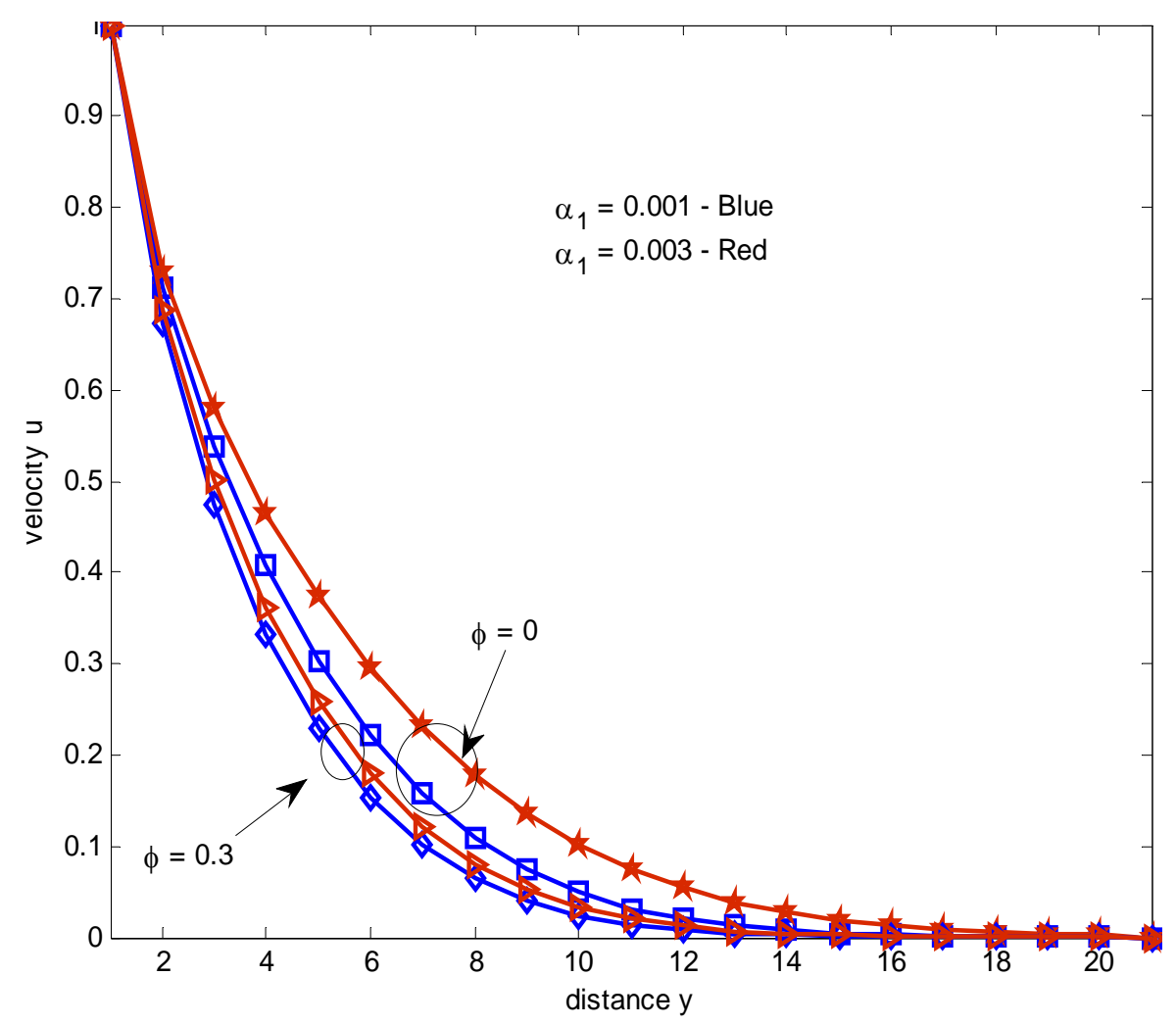

Fig.7. Velocity Profile for Fluid Phase $\left(E c=0.1, \alpha_{2}=0.025, \gamma=0.8, G r=0.2\right)$

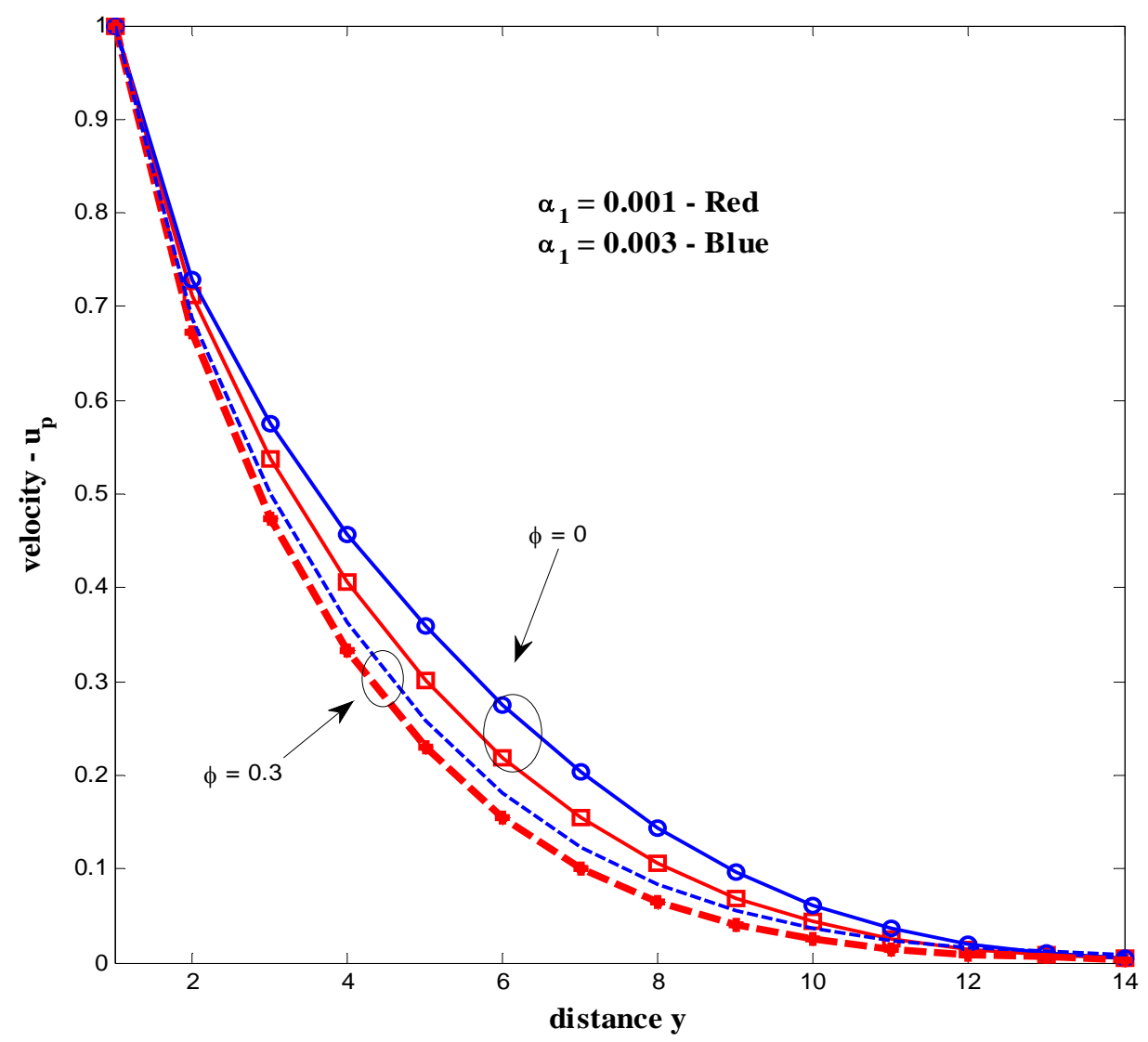

Fig.8. Velocity Profile for Dust Particle Phase $\left(E c=0.1, \alpha_{2}=0.025, \gamma=0.8, G r=0.2\right)$ 


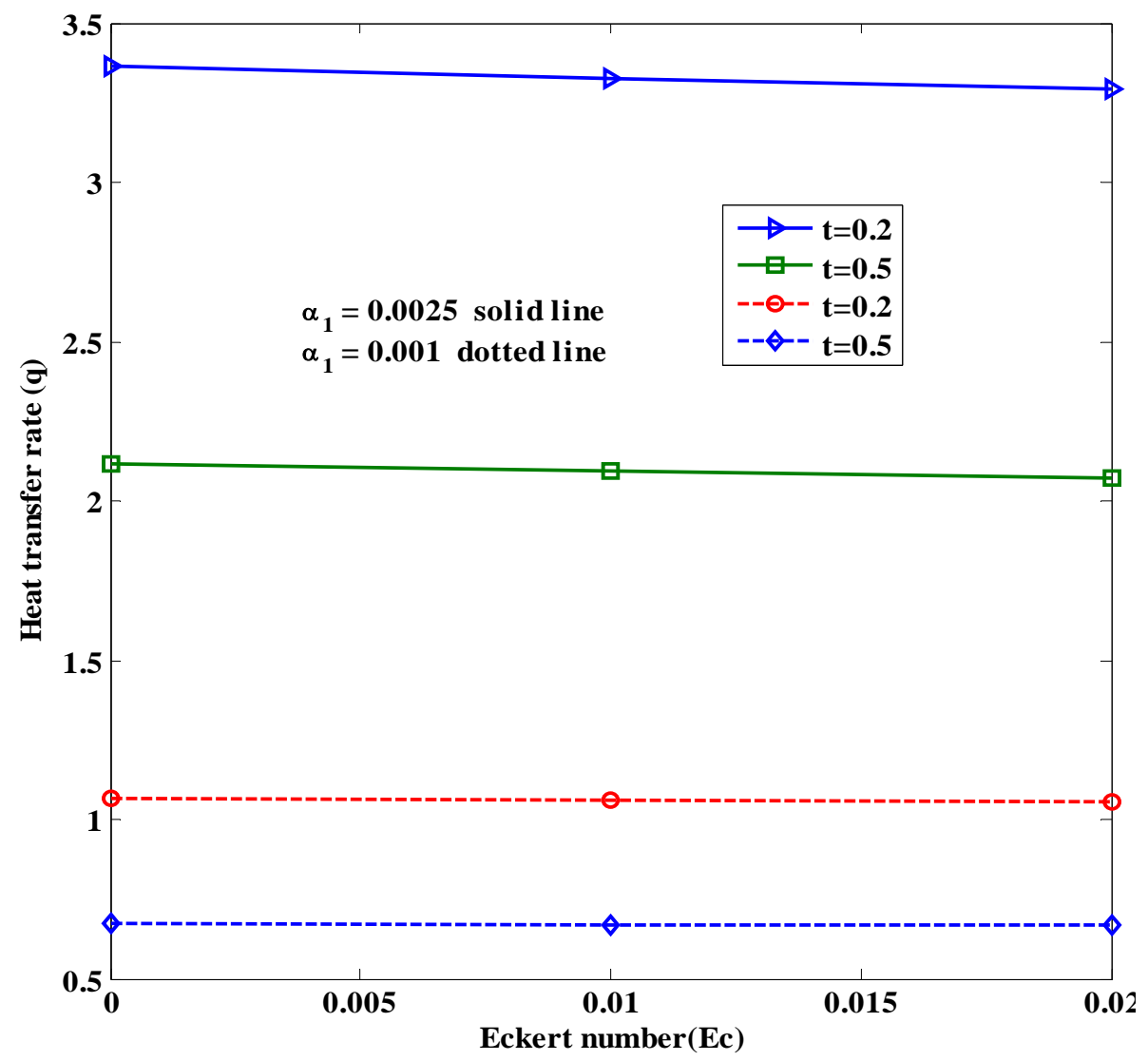

Fig.9. heat transfer rate against Eckert number $\left(\alpha_{2}=0.025, \gamma=0.8, G r=0.2\right)$

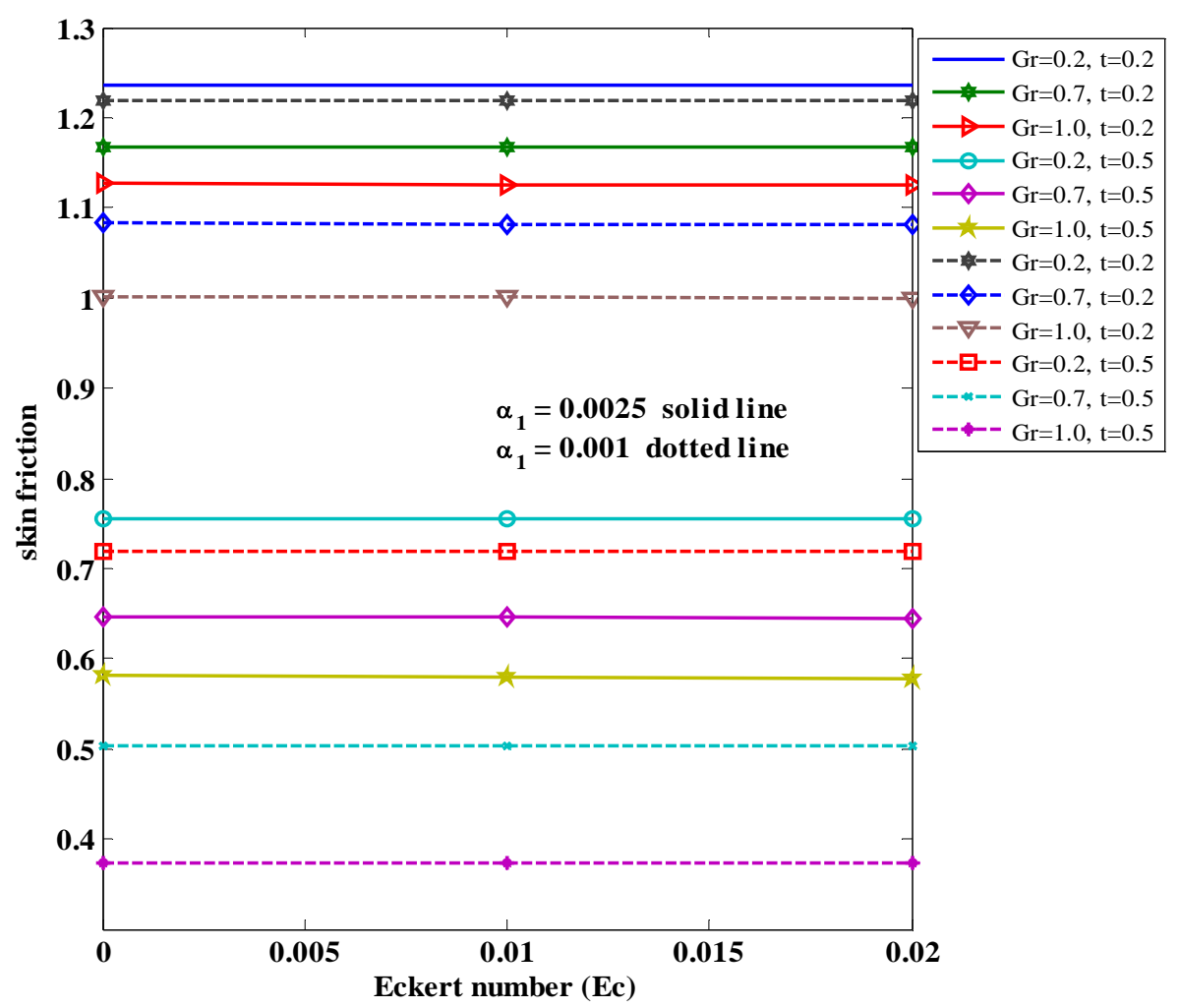

Fig.10. Skin Friction against Eckert number $\left(\alpha_{2}=0.025, \gamma=0.8, G r=0.2\right)$ 


\section{CONCLUSION}

A two-phase nanofluid viscous flow past an infinite vertical isothermal plate is considered. Copper $(\mathrm{Cu})$ and Alumina $\left(\mathrm{Al}_{2} \mathrm{O}_{3}\right)$ are submerged in a base fluid, Water $\left(\mathrm{H}_{2} 0\right)$. The coupled non-linear governing differential equations are solved by finite difference method. It is observed that due to viscous dissipative heat, there is a rise not only in velocity of fluid (nanofluid) but also in dust particle phase. Volume fraction increases the thermal boundary layer. With respect to Grashof number, it is noted that compared to fluid velocity, dust particle velocity is less in both the nanoparticles. Eckert number enhances the heat transfer rate in both phases.

\section{REFERENCES}

[1] L. Elliott, Unsteady laminar flow of gas near an infinite flat plate, Z. Angew, Math.Mech. 49, 647-652, 1969.

[2] L. J. Crane, Flow past a stretching plate, Zeitschrift f "ur Angewandte Mathematik und Physik ZAMP, 21(4), pp. 645-647, 1970.

[3] L. J. Grubka and K. M. Bobba, Heat transfer characteristics of a continuous stretching surface with variable temperature, Journal of Heat Transfer, 107(1), pp. 248-250, 1985.

[4] F.E. Marble, Dynamics of dusty gases, Ann Rev Fluid Mech., 2, pp.397-446, 1970.

[5] R. Cortell, A note on magneto-hydrodynamic flow of a power-law fluid over a stretching sheet, Applied Mathematics and Computation, 168(1), pp. 557-566, 2005.

[6] P. G. Saffman, On the stability of laminar flow of a dusty gas, Journal of Fluid Mechanics, 13, pp. 120-128, 1962.

[7] A.K.Singh, N.P. Singh, MHD flow of a dusty visco-elastic liquid through a porous medium between two inclined parallel plates, Proc Natl Acad Sci India,66(A), pp.143-50,1996.

[8] A. Chakrabarti, A.S. Gupta, Hydromagnetic flow and heat transfer over a stretching sheet, Q Appl Math, 37,pp.73-98, 1979.

[9] D.C. Dalal, N. Datta, S.K. Mukherjea, Unsteady natural convection of a dusty fluid in an infinite rectangular channel, Int. J. Heat Mass Transfer,41(3),pp.547-62, 1998.

[10] F.M. Allan, N. Qatanani, I. Barghouthi, M.K. Takatka, Dusty gas model of flow through naturally occurring porous media, App Math Comp, 21, pp.148:809, 2004.

[11] N. Sandeep, V. Sugunamma, Effect of inclined magnetic field on unsteady free convection flow of a dusty viscous fluid between two infinite flat plates filled by porous medium. Int J Appl Math Model,1, pp.16-33, 2013.

[12] O.D. Makinde, A. Aziz, Boundary layer flow of a nanofluid past a stretching sheet with convective boundary condition, Int J Ther. Sci, 50, pp. 26-32, 2011.

[13] F.M. Hady, F.M. Ibrahim, S.M. Abdel-Gaied, M.R. Eid, Radiation effect on viscous flow of a nanofluid and heat transfer over a nonlinearly stretching sheet. Nanoscale Res Lett, 7, pp.299-308, 2012.

[14] V.Venkataraman and K. Kannan, Numerical solution of Stokes problem for free convection effects in dissipative dusty medium, Int.J. Mathematics and Mathematical sciences, 72, pp.3975-3988, 2004.

[15] H.F.Oztop, E. Abu-Nada, Numerical study of natural convection in partially heated rectangular enclosures filled with nanofluids, Int.J. Heat and Fluid Flow, 29(5),pp.1326-36,2008.

\section{AUTHOR PROFILE}

Author1: Dr.D.R.V.S.R.K. Sastry, received his Ph.D degree from Andhra University, Visakhapatnam in 2013. He is working in the Department of Mathematics, SASTRA University, Thanjavur. He has 18 years of teaching experience. His current research areas include Computational fluid dynamics.

Author2: Dr.V.Venkataraman obtained his Master's degrees M.Sc., M.Phil., from Bharathidasan University and Ph.D from SASTRA University. He is working in the Department of Mathematics, SASTRA University, Thanjavur, since 2000. His current research areas include Computational Fluid Dynamics, Optimization and Differential Equations.

Author3: Dr.K.Kannan, received his Ph.D degree from Allagappa Univeristy, Karaikkudi, in 2003. He published around 78 papers in referred journals. He has 35 years of teaching experience. He guided 7 Ph.D students so far. He is currently working as Dean, School of Humanities and Sciences in SASTRA University. His research areas of interest are Computational Fluid Dynamics, Image processing, Algorithms, Wavelet Transforms, Hyper-graphs, Artificial neural Network, Stochastic process.

Author4: M. Srinivasu, obtained his M. Sc in Andhra University, Visakhapatnam. He is currently working in Aditya College of Engineering, Surampalem, Andhra Pradesh. He has 15 years of teaching experience. 